\title{
Trabalho em equipe: percepção interprofissional de uma clínica pediátrica
}

\author{
Teamwork: interprofessional perception of a pediatric clinic \\ Trabajo en equipo: percepción interprofesional de una clínica pediátrica
}

\author{
Marina Freire Nunes ${ }^{l}$ \\ Luciana Rodrigues Wovst \\ Sebastião Benício da Costa Neto \\ Hospital das Clínicas da Universidade Federal de Goiás
}

\begin{abstract}
Resumo
As instituições de saúde devem se basear em novos parâmetros relacionais para o melhor atendimento ao paciente, a qualidade de vida e o trabalho em equipe. Assim, objetiva-se descrever e analisar a percepção de profissionais de saúde sobre o trabalho no cotidiano da Clínica Pediátrica (CP), bem como as barreiras e os facilitadores da relação entre as equipes. Participaram 12 profissionais, com formação técnica e superior em distintas categorias. Utilizaram-se um questionário sociodemográfico, uma Escala de Percepção Interprofissional e um roteiro de entrevista semi-estruturada, nos meses de setembro e outubro de 2012. A equipe avaliou seus membros como competentes, sendo os da área de humanas com melhor percepção interprofissional. Apesar das dificuldades, diversos fatores relacionais contribuíram para o desenvolvimento e qualidade do trabalho prestado na CP. Concluise que a percepção interprofissional da equipe da pediatria foi positiva.

Palavras-chave: Trabalho em equipe; Equipe multiprofissional; Percepção interprofissional.
\end{abstract}

\begin{abstract}
Health institutions should be based on new parameters related to develop a better patient care, life quality and teamwork. Therefore, our research intends to describe and analyze the perception of health professionals on the routine in a Pediatric Clinic (PC), as well as the difficulties and the factors that assist the relations among the staff. We worked, as part of our research, with twelve professionals with distinct categories technical and graduation formation. A social demographic questionnaire, a Scale of Interprofessional Perception and a script of semistructured interview were used in September and October of 2012. The staff rated its members as proficient, being the humanities area rated as the best interprofessional perception. Despite of difficulties, some relations factors contributed for the development and quality of the work provided at a PC. As conclusion, the interprofessional perception of the pediatric staff was taken by the members as positive.

Key-words: Teamwork; Multiprofessional staff; Interprofessional perception.
\end{abstract}

\section{Resumen}

Las instituciones de salud deben basarse en nuevos parámetros relacionales para la mejor atención al paciente, la calidad de vida y el trabajo en equipo. Así, se objetiva describir y analizar la percepción de profesionales de salud sobre el trabajo diario de la Clínica Pediátrica (CP), bien como las barreras y los facilitadores de la relación entre los equipos. Han participado 12 profesionales, con formación técnica y superior en distintas categorías. Se utilizaron un cuestionario socio demográfico, una Escala de Percepción Interprofesional y una guía de entrevista semiestructurada, en los meses de septiembre y octubre de 2012. El equipo ha evaluado a sus miembros competentes, siendo los del área de humanas con mejor percepción interprofesional. A pesar de las dificultades, varios factores relacionales contribuyeron para el desarrollo y calidad del trabajo prestado en la CP. Se concluye que la percepción interprofesional del equipo de la pediatría fue positiva.

Palabras-clave: Trabajo en equipo; Equipo multiprofesional; Percepción interprofesional.

\section{INTRODUÇÃO}

A área da saúde passou por uma mudança de paradigma, no qual o conceito cartesiano, ainda hegemônico, caracterizado pelo reducionismo e fragmentação da saúde, abriu espaço a uma concepção mais abrangente, na qual se valoriza a atuação intersetorial no nível dos determinantes da

Endereço 1: Rua S-5 n. 157 apto 1202 Ed. Danville, Setor Bela Vista, Goiânia, Goiás. CEP 74823-460.

Endereço 2: Rua Teresina, n. 419, Apto, 1601, Setor Alto da Glória, Goiânia - GO. CEP 74815-715. saúde (Vilela \& Mendes, 2000).

Essa transição estabelece uma revisão acerca do papel classicamente atribuído e efetivamente exercido pelos profissionais de saúde, pautado no modelo biomédico, gerando alterações na forma de compreender e constituir o trabalho no campo da atividade humana no ambiente hospitalar (Queiroz \& Araújo, 2009).

AResoluçãon ${ }^{\circ} 287$, de 1998, do Conselho Nacional de Saúde (BRASIL, 1998) ampliou a compreensão do conceito de saúde/doença, ressaltando a importância das ações interdisciplinares no âmbito da saúde e 
reconheceu que as ações realizadas pelos diferentes profissionais de nível superior constituem um avanço no que tange à concepção de saúde e à integralidade da atenção.

Com isso, a saúde deve ser pensada dentro de uma perspectiva global, integrada às condições de vida e trabalho da comunidade, entendendo que a conjunção e articulação de diferentes conhecimentos e ações, envolvendo um trabalho em equipe, torna-se condição essencial à eficácia das práticas de saúde, humanizando sua atuação.

Segundo Peduzzi (2001), equipe consiste em uma ação integrada, regida por normas consensuais, baseada na intersubjetividade da compreensão e do reconhecimento mútuo, isenta de coerção. A equipe possui um desempenho coletivo, com associação gerencial de habilidade e talentos individuais em uma habilidade coletiva, produzindo atividades e atuações de maneira mais eficiente e efetiva, na qual o resultado é maior do que a soma das partes individuais (Abreu, Munari, Queiroz \& Fernandes, 2005).

Conforme Queiroz e Araújo (2009) apresentam, a atuação do trabalho em equipe visa um atendimento integral, fortalecendo a autonomia e a qualidade de vida do paciente. Todavia, para que a assistência prestada favoreça a compreensão do paciente acerca do seu estado de saúde e estimule o exercício de sua autonomia, faz-se necessária uma melhor integração da equipe, bem como uma atuação articulada e coerente. Para a integração da equipe, é de vital importância à clarificação, entre todos os seus integrantes, das suas atribuições, papéis, limitações e possibilidade de intervenção.

Neste sentido, pode-se assegurar que um bom relacionamento entre equipe-paciente-família favorece todos os envolvidos, visto que a inclusão do paciente e da família no trabalho da equipe, contribuindo para uma postura ativa e participativa, proporciona maior confiança, segurança, acolhimento do paciente, refletindo em um melhor enfrentamento do processo de saúde-doença (Santos \& Sebastiani, 1996).

O trabalho em equipe pode adquirir várias configurações, de acordo com o seu modo de funcionamento, tais como pluridisciplinar, multidisciplinar, interdisciplinar e transdisciplinar. O foco do estudo é na equipe multiprofissional, de caráter interdisciplinar.

A atuação multiprofissional se configura como um trabalho coletivo organizado por profissionais de diversas categorias, cuja contribuição individual ressoa no produto final, atendendo às particularidades de cada área na solução de problemas diagnosticados em conjunto pelos seus membros (Pereira \& Harris, 1976). A interação da equipe de saúde propicia aos profissionais um enriquecimento de sua prática, aprofundamento do saber, permitindo a ampliação do conhecimento por meio da contribuição das diferentes categorias profissionais, potencializando a atuação da equipe.

Galván (in Gomes e Deslandes , 2007) expõem que a interdisciplinaridade leva a uma reflexão profunda a respeito do conceito de ciência, possibilitando o resgate da unidade do seu objeto de estudo, no caso o paciente.

Neste sentido, a equipe interdisciplinar trabalha com foco nas necessidades do paciente, favorecendo a integração dos profissionais de saúde, com o intuito de satisfazer as necessidades globais da pessoa, visando seu bem-estar. As dificuldades e situações inesperadas vivenciadas pelos usuários do serviço de saúde nos hospitais ressoam no trabalho da equipe, demonstrando que uma única categoria profissional não consegue abarcar todos os fatores intrínsecos ao processo de saúde e doença e à hospitalização (Fossi \& Guareschi, 2004).

Santo e Sebastiani (1996) destacam que as disputas e as divergências estão vinculadas a uma formação profissional geradora de sentimentos onipotentes, centralizadores e individualistas, os quais estimulam as barreiras da relação entre os profissionais e, consequentemente, acabam interferindo nas relações da equipe com os pacientes e seus familiares.

O desconhecimento do saber do outro propicia a hegemonia de determinadas profissões sobre as outras, favorecendo tanto a desvalorização, quanto a subordinação indevida, dentro equipe (Fossi \& Guareschi, 2004). Podem-se constatar, também, dificuldades institucionais expressas na falta de espaço e horários comuns para reuniões, comprometendo a comunicação da equipe.

Abreu et al.(2005) expõem em seus estudos que o fator que mais interfere na ação integrada, acarretando insegurança e insatisfação, é o relacionamento interprofissional. $\mathrm{O}$ respeito e a comunicação se configuram como fundamentais para um processo de trabalho, atuando na amenização dos conflitos.

É importante ressaltar, entretanto, que a configuração de trabalho em equipe ainda encontrase em construção, possuindo inúmeras limitações e barreiras. (Queiroz \&Araújo, 2009).

Leite e Vila (2005) frisam que a equipe multiprofissional vivencia momentos estressantes, tais como a dificuldade frente à morte e aos recursos materiais e humanos insuficientes. Em suma, esse cenário promove tensões e interfere negativamente na qualidade da assistência prestada ao usuário. Os autores (2005) ressaltam, ainda, que a delimitação dos papeis profissionais dos membros da equipe de saúde favorecem a diminuição da ansiedade e estimula o profissional a se responsabilizar pelas suas atitudes. Desse modo, deve-se incentivar o desenvolvimento do relacionamento da equipe por meio da valorização do diálogo construtivo, da honestidade, da amizade e do respeito mútuo, bem como impulsionar a motivação do grupo para a construção de uma equipe 
articulada, harmoniosa, humana e comprometida com a assistência de qualidade.

Fazendo referência a Silva, Scapin e Batista (2001), a convivência e a formação interprofissional alteram atitudes e percepções negativas entre os membros da equipe de saúde prevenindo falhas na confiança e na comunicação.

Considerando o exposto é preciso atentar-se, ainda, às dificuldades específicas do trabalho em uma clínica pediátrica, já que essas ampliam os obstáculos para o trabalho em equipe. Na Clínica Pediátrica a equipe de saúde vivencia o adoecimento da criança, a mudança de sua estrutura familiar e suas consequentes adaptações ao processo saúde-doença. Com isso, a atuação da equipe não se restringe ao paciente, no caso a criança, envolvendo a família como um todo.

A verdadeira terapêutica é aquela que zela pela criança como um todo e não simplesmente foca no seu sintoma, no seu quadro clínico (Mello Filho, 1992). Neste sentido, pode-se dizer que a participação de uma equipe multiprofissional com suas especificidades e particularidades no cuidado com a criança e sua família são imprescindíveis.

Castro e Piccinini (2002) afirmam que é elevada a prevalência de doenças crônicas em crianças com implicações em seu desenvolvimento e em suas relações familiares e sociais. As crianças ficam sujeitas a recorrentes internações, passando pela realização de diversos exames e procedimentos invasivos.

Além disso, o apoio da equipe de saúde à criança e à família é extremamente importante para o enfrentamento do processo de doença, internação e tratamento. A equipe passa a integrar esse novo sistema e suas relações passam a influenciar o modo de enfrentamento dessa nova realidade, que implica em mudanças nas rotinas e em adaptações psicológicas e existenciais que envolvem o paciente, seus familiares e a equipe de saúde.

Com isso, a experiência profissional e o contato contínuo com o ambiente de uma clínica pediátrica de um hospital escola em Goiânia subsidiou o interesse pela realização desse estudo. A observação tem mostrado que a realidade dessa clínica, caracterizada pelo cuidado de crianças com doenças crônicas, é permeada por vários sentimentos e emoções e que a rotina exige uma excelente capacitação técnicocientifica e preparo para lidar com situações de urgência/emergência, de perdas, de instabilidades e falta de estrutura.

Assim, neste estudo, busca-se descrever e analisar a percepção de profissionais de saúde sobre o trabalho multidisciplinar no cotidiano da clínica pediátrica de um hospital universitário público, bem como as barreiras e os facilitadores da relação entre os profissionais, partindo dos seguintes objetivos específicos: $1^{\circ}$ ) verificar quais são as percepções interprofissionais em relação às diversas categorias profissionais da Clínica Pediátrica; $2^{\circ}$ ) comparar as percepções interprofissionais de profissionais de formação de nível superior com os de nível técnico no contexto da Clínica Pediátrica; $3^{\circ}$ ) compreender e descrever as atitudes e os valores relacionados ao trabalho da equipe de saúde dos profissionais do contexto da Clínica Pediátrica do; $4^{\circ}$ ) identificar quais barreiras interferem no trabalho em equipe na Clínica Pediátrica; e $5^{\circ}$ ) reconhecer quais facilitadores auxiliam nas relações da equipe da Clínica Pediátrica.

\section{METODOLOGIA}

\section{PARTICIPANTES}

Participaram do estudo 12 profissionais, todos do sexo feminino, que compõem a equipe de saúde da clínica pediátrica, com formação técnica e superior em distintas categorias profissionais, vinculadas há mais de 12 meses à Pediatria do hospital universitário público, que não estiveram de licença nos últimos seis meses e que, também, assinaram o Termo de Consentimento Livre e Esclarecido.

Entre as profissionais 2 são médicas, 7 são enfermeiras, 2 são técnicas de enfermagem e 1 é psicóloga. Com relação a idade, seis possuem entre 41 e 50 anos, 3 (três) entre 31 e 40 anos e 3 (três) mais de 50 anos. Cinco delas são casadas, quatro são solteiras, duas divorciados e uma viúva. Em relação à religião, 7 (sete) são católicas, 3 (três) evangélicas e 2 (duas) espíritas. Se tratando do grau de escolaridade, 6 (seis) das profissionais possuem especialização, sendo as outras 6 (seis) divididas, igualmente, entre profissionais que possuem formação técnica, graduação e mestrado. Destas profissionais, 5 (cinco) possuem entre 16 e 20 anos de formação profissional, 3 (três) entre 11 e 15 anos, 2 (duas) mais de 21 anos e 1(uma) possuem entre 1 e 5 anos e 6 e 10 anos, sendo que 6 (seis) possuem entre 16 e 20 anos de atuação na clínica pediátrica, 4 (quatro) entre 11 e 15 anos e 1 (uma) entre 1 e 5 anos e mais de 21 anos. A amostra das participantes foi definida de acordo com o critério de conveniência, não probabilístico (Cozby, 2006), considerando a demanda dos profissionais da equipe dentre os critérios de inclusão, a disponibilidade dos mesmos, a conveniência e a realização do estudo na data acadêmica exigida. Trata-se, assim, de um trabalho de classificação, descritivo, exploratório e qualitativo, visto que os resultados não serão generalizados.

\section{RESPONSABILIDADE ÉTICA}

O estudo foi elaborado tendo em vista o que preconiza a Resolução 196/96, do Ministério da Saúde (BRASIL, 1996), sobre pesquisa envolvendo seres humanos, resguardando, portanto, a responsabilidade ética dos pesquisadores. Iniciou-se a coleta de dados após o consentimento institucional e o parecer favorável do Comitê de Ética em Pesquisa do Hospital das Clínicas da Universidade Federal de Goiás. 


\section{INSTRUMENTOS}

O estudo propôs a utilização de um questionário sócio demográfico, uma Escala de Percepção Interprofissional - EPI (Ducanis \& Golin, 1979) e um roteiro de entrevista semi-estruturada.

A Escala de Percepção Interprofissional EPI apresenta a possibilidade de uma avaliação interprofissional em três níveis: a percepção do respondente sobre as categorias profissionais (A), a percepção do respondente acerca da percepção do profissional examinado (B) e a percepção do profissional examinado quanto à percepção das demais profissões sobre a sua percepção $(\mathrm{C})$.

\section{PROCEDIMENTOS}

Os instrumentos foram respondidos no consultório multiprofissional da pediatria, devidamente preparados para o processo de coleta de dados e adotando a seguinte ordem: questionário sócio demográfico, questionário de escala de percepção interprofissional e roteiro de entrevista semi-estruturada de caracterização do ambiente profissional/relacional da pediatria. A coleta de dados teve duração média de 30 minutos e foi realizada durante os meses de setembro e outubro de 2012.

\section{ANÁLISE DE DADOS}

O questionário sócio demográfico foi avaliado por meio de estatística descritiva simples (frequência e média). Já o questionário de escala de percepção interprofissional, teve consideradas apenas as respostas verdadeiras referente às questões dos dois primeiros níveis de avaliação (A e B), sendo comparados, considerando que uma divergência significativa corresponde a uma variação de $15 \%$ de diferença entre as duas. $\mathrm{O}$ terceiro nível $\mathrm{C}$ não será analisado nesse estudo. E no roteiro de entrevista semi-estruturada de caracterização do ambiente profissional/relacional na pediatria, os dados foram submetidos ao processo de análise de conteúdo, especificamente, na modalidade da temática, tal como proposta por Bardin (2008).

\section{RESULTADOS E DISCUSSÃO}

A partir dos dados da Escala de Percepção Interprofissional-EPI, elegeram-se nove categorias de análise (descritas nos gráficos de 1 a 15) com o intuito de verificar quais são as percepções interprofissionais em relação às diversas categorias da clínica pediátrica de um hospital universitário público.

O Gráfico 1 apresenta a percepção dos profissionais - médicos (M), da área biomédica (B) e da área de humanas $(\mathrm{H})$ - da Clínica Pediátrica em relação a competência profissional da equipe.

Os dados evidenciam que as médicas $(\mathrm{M} / \mathrm{N}=2)$ e a profissional da área de humanas $(\mathrm{H} / \mathrm{N}=1)$ avaliam toda a equipe como competente (100\%), assim como a maioria das profissionais da área biomédica (B / $\mathrm{N}=9$ ), $\mathrm{M}=89 \%, \mathrm{~B}=89 \%$ e $\mathrm{H}=67 \%$, não havendo divergência significativa entre os dois níveis de avaliação (A e B). Com isso, os dados demonstram que todos os profissionais participantes avaliam os integrantes da equipe de saúde da Clínica Pediátrica como competentes. Esta percepção dos profissionais pode estar relacionada à transição do modelo de saúde ainda presente nas instituições hospitalares, bem como ao processo de construção do trabalho em equipe multiprofissional no contexto hospitalar.

Embora as instituições hospitalares estejam abertas a configurações de equipe multiprofissionais de saúde, o Gráfico 2 demonstra a percepção dos profissionais da equipe da Clínica Pediátrica em relação a pouca autonomia profissional.

De acordo com os dados, pode-se constatar que a maioria dos profissionais da equipe avaliam os profissionais da área biomédica (B) e da área de humanas $(\mathrm{H})$ como pouco autônomos, o que não

Gráfico 1

Percepção das profissionais da Clínica Pediátrica em relação a competência profissional

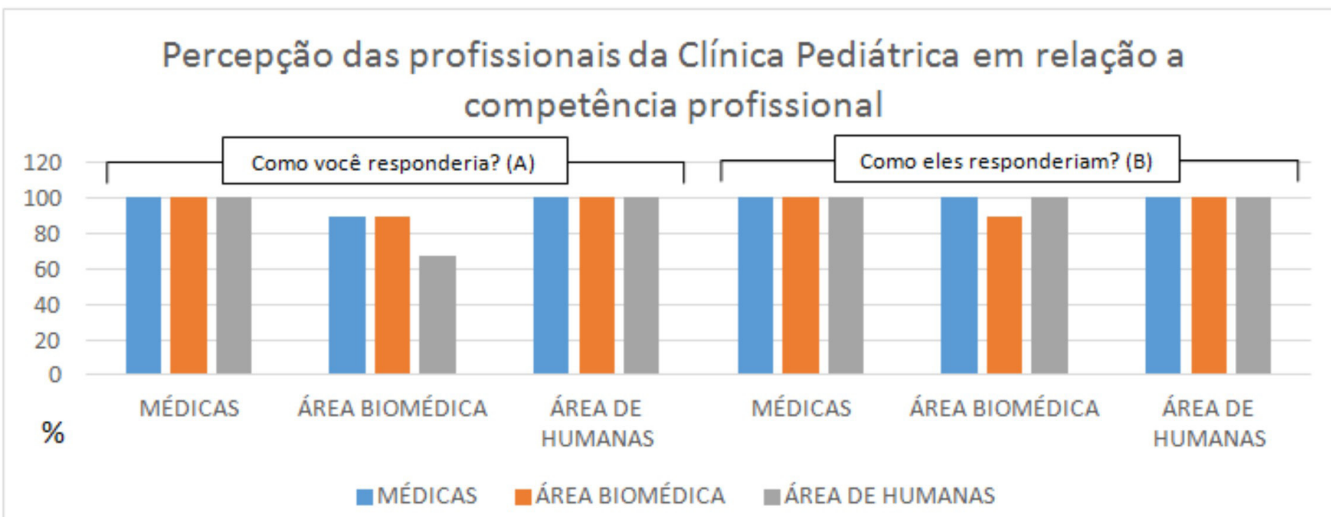


Gráfico 2

Percepção das profissionais da Clínica Pediátrica em relação a pouca autonomia profissional

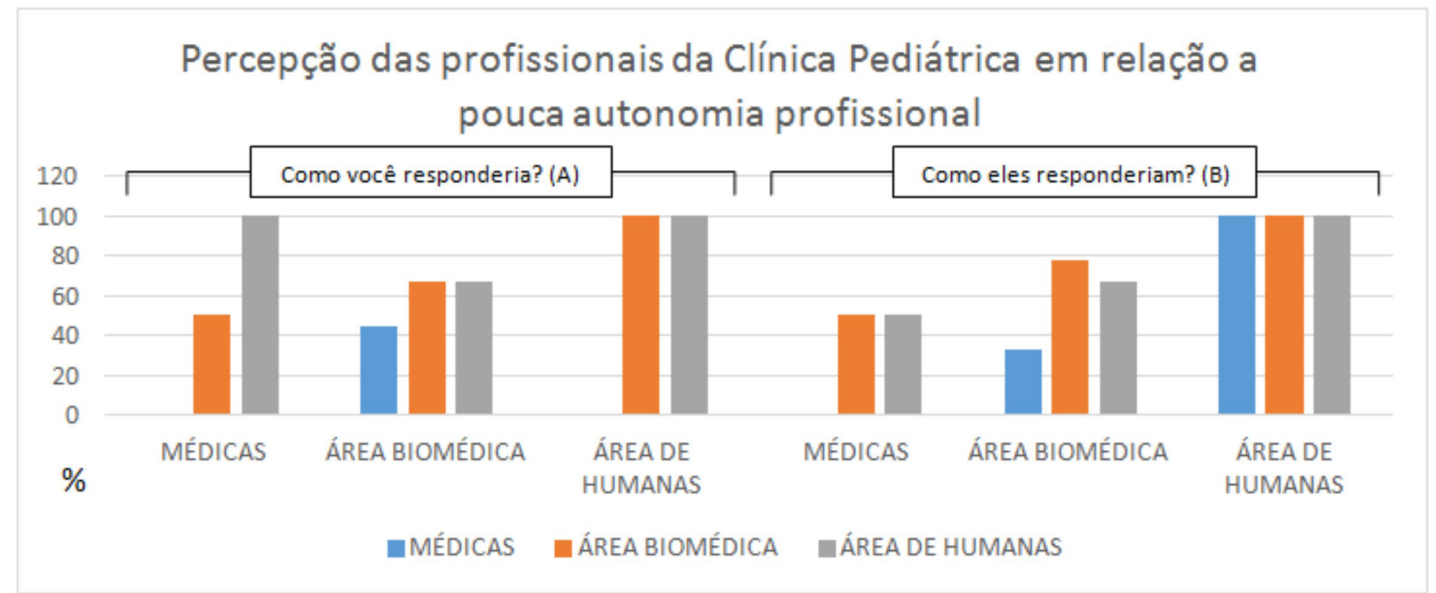

Gráfico 3

Percepção das profissionais da Clínica Pediátrica acerca da compreensão das suas capacidades profissionais da equipe.

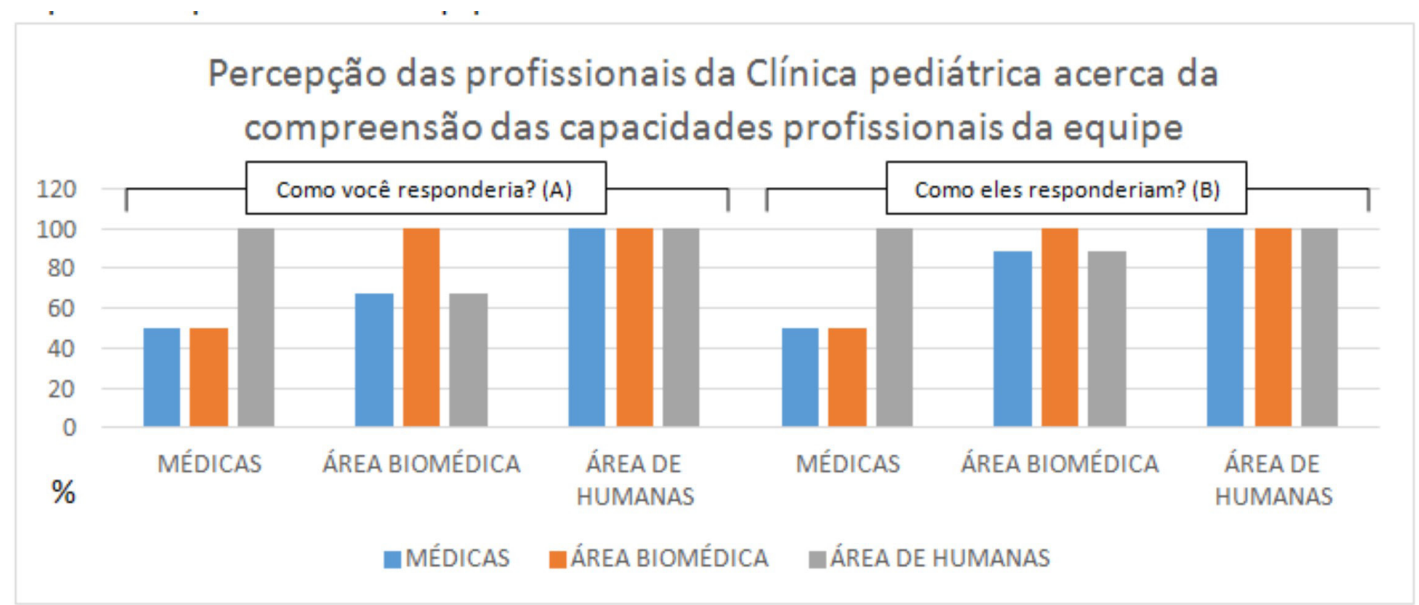

ocorre em relação aos médicos. No entanto, verificase uma discrepância entre o nível de avaliação A e o $B$ em relação a percepção dos profissionais da área de humanas em relação a pouca autonomia dos médicos $(0 \%-100 \%)$

Além disso, o Gráfico 3 evidencia a percepção dos profissionais da Clínica Pediátrica acerca da compreensão das capacidades profissionais da equipe.

Os dados demonstram que os profissionais da área biomédica e da área de humanas possuem uma melhor compreensão em relação as capacidades profissionais da equipe. Neste sentido, quanto maior a autonomia, menor a interação entre os membros da equipe, já que a configuração de trabalho em equipe na Pediatria, ainda, encontra-se em construção, possuindo algumas limitações e barreiras.

Assim, como apresenta Wallin e Filho (2007), a inserção de outras categorias profissionais no hospital acarreta o surgimento de questões referentes ao trabalho em equipe, que envolvem dificuldades quanto à indefinição de papeis, de fronteiras profissionais e decorrentes do poder de decisão, competitividade interprofissional, hierarquização das profissões e barreiras no entrosamento interprofissional. Há, também, uma tendência dos profissionais em satisfazer suas aspirações e manter sua autonomia, em detrimento da colaboração profissional.

Essa tendência também é evidente em relação à percepção interprofissional quanto à preocupação com o bem estar do paciente exposta no Gráfico 4

Os dados apresentam que os médicos avaliam a equipe como preocupada com o bem estar dos pacientes, o que não ocorre na percepção dos outros profissionais, exceto em relação aos profissionais da área de humanas, uma vez que há uma discrepância significativa em relação à percepção dos profissionais da área biomédica e da área de humanas nos dois níveis de avaliação A e B referentes aos profissionais médicos $(22 \%-89 \%)$ e aos profissionais médicos $(0 \%-100 \%)$ e da área de biomédicas $(0 \%-100 \%)$, respectivamente, o que não acontece na percepção dos médicos. 
Gráfico 4

Percepção das profissionais da clínica pediátrica acerca da preocupação da equipe em relação ao bem estar dos pacientes

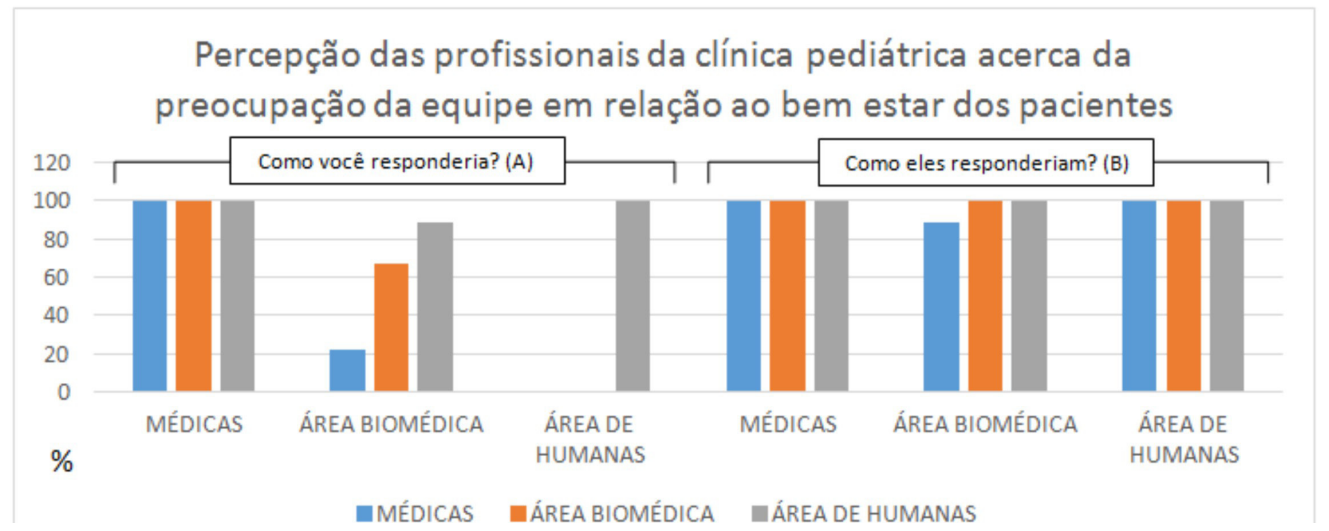

Gráfico 5

Percepção das profissionais da Clínica Pediátrica acerca da invasão do terreno profissional na equipe

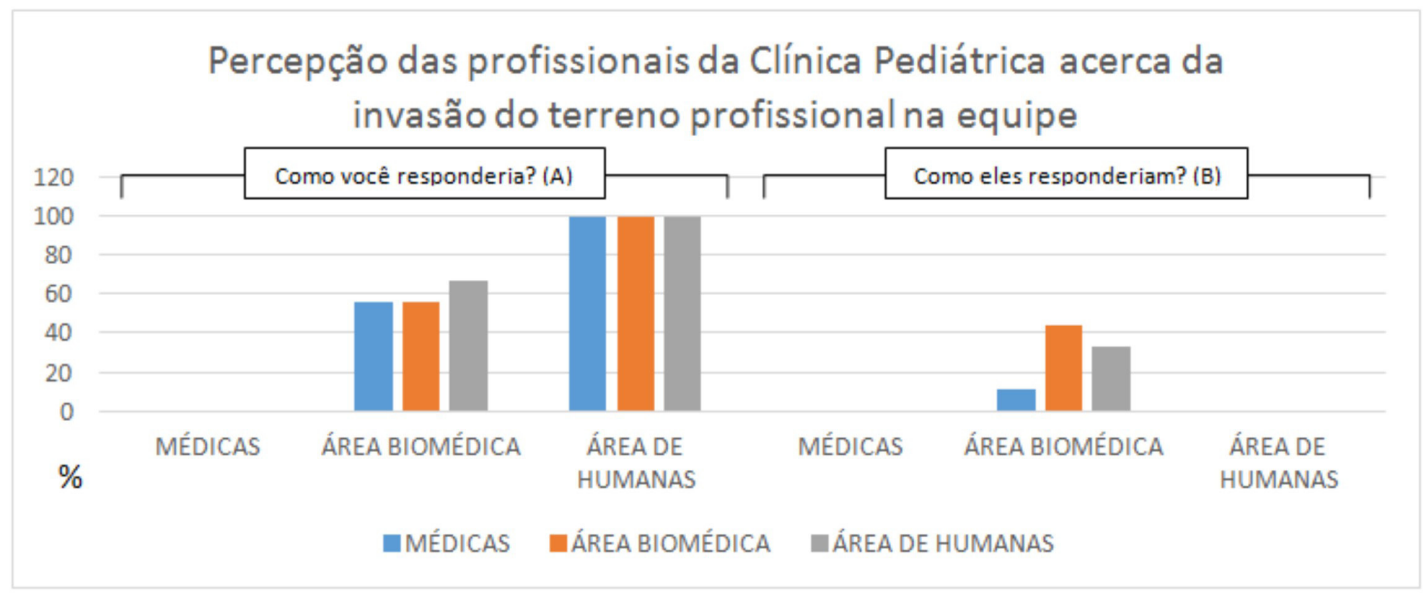

Pode-se constatar, ainda, que umas das barreiras existentes entre a equipe multiprofissional da Clínica Pediátrica está evidenciada no Gráfico 5, que aborda a percepção dos profissionais acerca da invasão do terreno profissional.

Os dados demonstram que todos os médicos (M) desconsideram a invasão profissional dentro da equipe. No entanto, tanto os profissionais da área biomédica (B) quanto os profissionais da área de humanas $(\mathrm{H})$ apresentam uma discordância entre os dois níveis de avaliação A e B. De acordo com os profissionais da área biomédica as dissonâncias ocorrem em relação aos profissionais médicos $(56 \%$ - 11\%) e aos profissionais da área de humanas (67\% - 33\%). Já a profissional da área de humanas expõe a divergência em relação a todos os profissionais $(100 \%-0 \%)$.

Nestecaso, faz-senecessárioumamaiorvalorização dos profissionais e uma melhor delimitação das suas capacidades profissionais. Assim, Leite e Vila (2005) (2005) ressaltam a importância da delimitação dos papeis profissionais dos membros da equipe de saúde, o que favorece a diminuição da ansiedade e estimula o profissional a se responsabilizar pelas suas atitudes.

O Gráfico 6 aborda a percepção dos profissionais da Clínica Pediátrica a respeito da postura ética dos profissionais da equipe.

De acordo com os dados, os médicos (M) avaliam a equipe como ética, não havendo uma divergência tão significativa entre os dois níveis de avaliação A e B. Entretanto, os profissionais da área biomédica (B) e da área de humanas $(\mathrm{H})$ expõem uma dissonância entre os dois níveis de avaliação A e B referentes aos médicos $(22 \%$ - $100 \%$ e $0 \%$ - 100\%), aos profissionais da área biomédica $(44 \%$ - 67\% e $0 \%-100 \%)$ e aos profissionais da área de humanas $(78 \%$ - 100\% e $0 \%$ - 100\%), respectivamente, sendo a área de humanas a com melhor avaliação quanto a ética profissional.

Já os Gráficos 7 e 8 abordam a percepção dos profissionais da Clínica Pediátrica acerca das expectativas da equipe frente suas profissões e a percepção quanto ao status profissional em relação 
Gráfico 6

Percepção das profissionais da Clínica Pediátrica em relação à postura ética dos profissionais da equipe

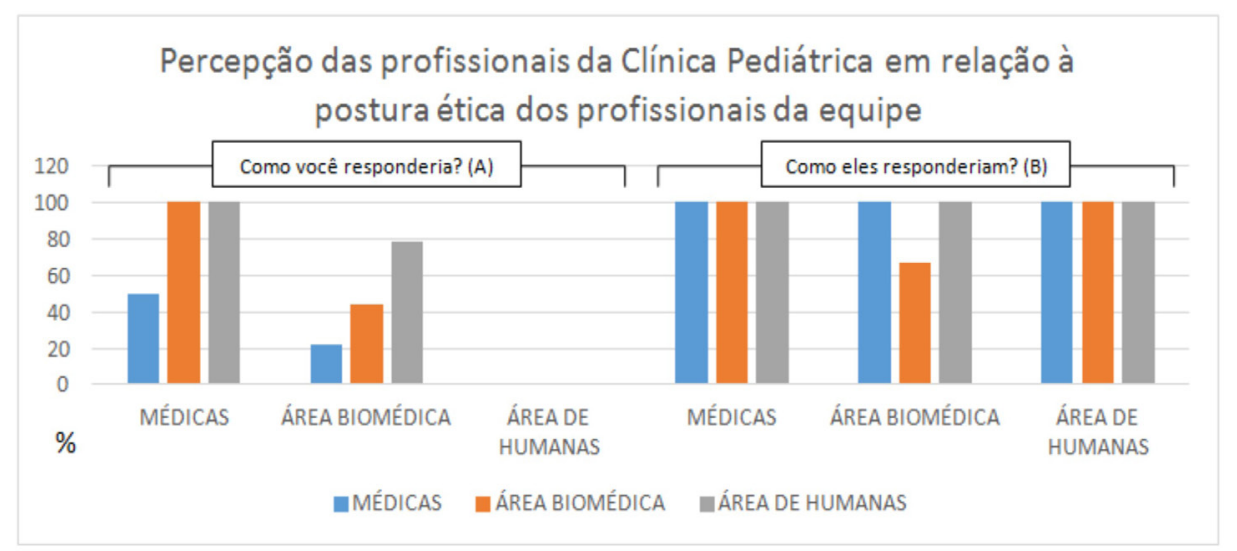

Gráfico 7

Percepção das profissionais da Clínica Pediátrica em relação à expectativa diante da sua profissão

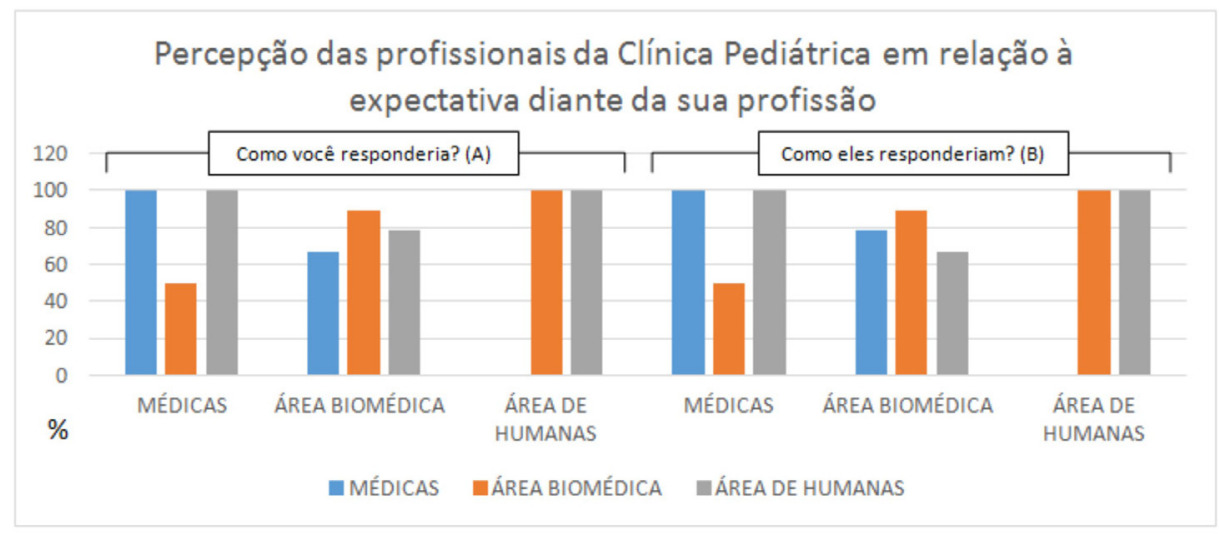

Gráfico 8

Percepção das profissionais da Clínica Pediátrica acerca do status profissionais em relação aos profissionais da equipe

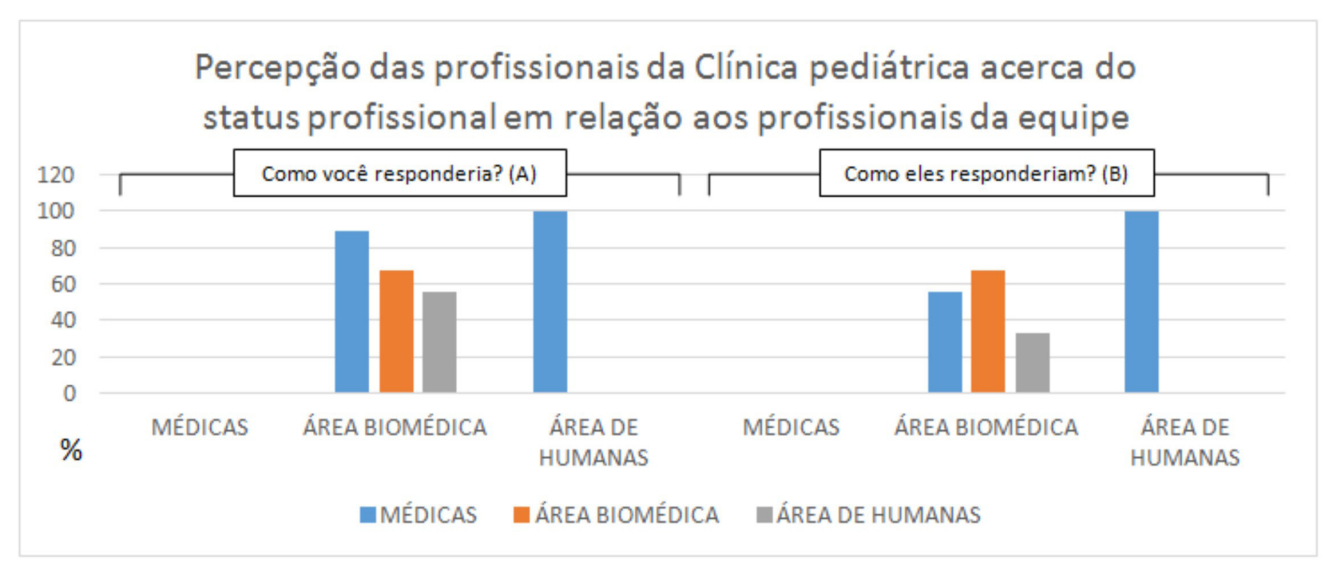

aos profissionais da equipe, respectivamente.

De forma geral, os dados apresentam uma boa expectativa da equipe frente às suas profissões, não havendo divergências significativas quando comparados os dois níveis de avaliação A e B. Apenas os profissionais da área de humanas $(\mathrm{H})$ percebem os médicos com nenhuma expectativa diante das suas profissões.
Nos dados presentes no Gráfico 8, pode-se constatar que os profissionais da área biomédica (B) e da área de humanas $(\mathrm{H})$ percebem que os profissionais médicos possuem um maior status profissional quando comparados aos da sua área profissional, o que não ocorre na percepção dos médicos (M)..

O Gráfico 9 expõe a percepção dos profissionais da Clínica Pediátrica acerca da postura defensiva 
Gráfico 9

Percepção das profissionais da Clínica Pediátrica acerca da postura defensiva em relação às regalias profissionais deles

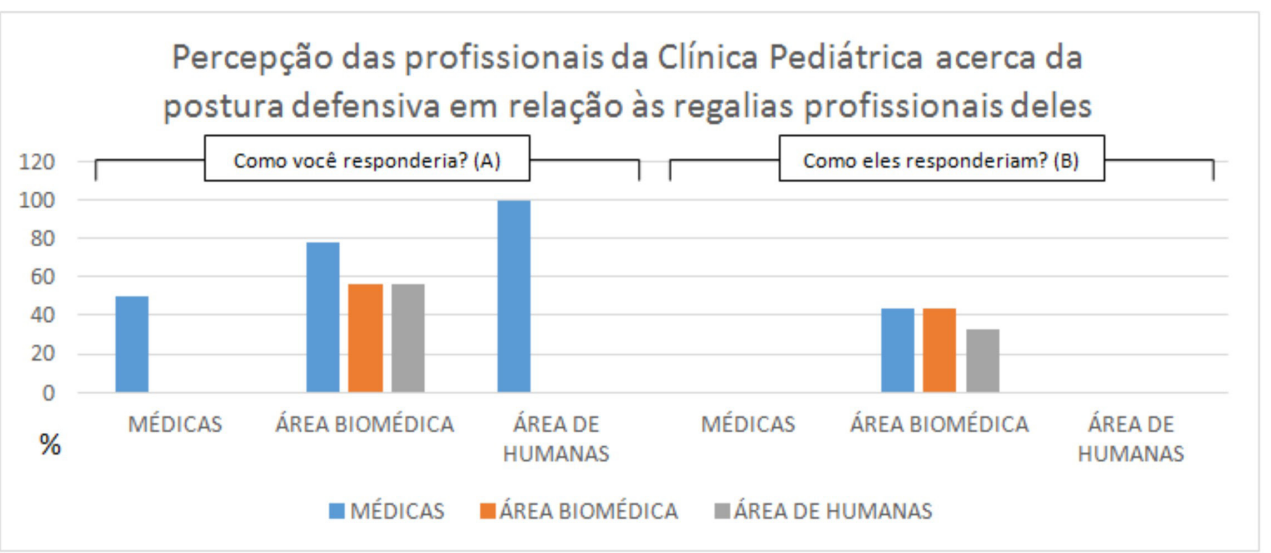

Gráfico 10

Percepção das profissionais da Clínica Pediátrica em relação à confiança do seu julgamento profissional

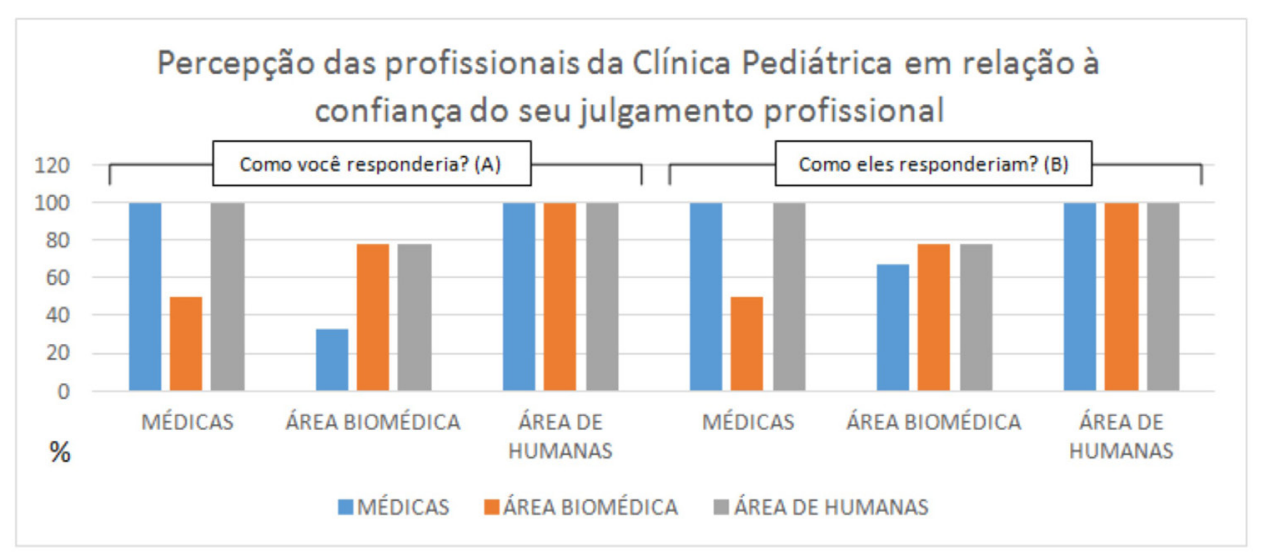

adotada pelos profissionais frente suas regalias profissionais.

Os dados evidenciam que, de acordo com a maioria dos profissionais das três categorias criadas, os médicos (M) possuem postura defensiva. Neste sentido, há discrepância entre os dois níveis de avaliação A e B: médicos (50\% - 0\%), profissionais da área biomédica $(78 \%$ - 44\%) e da área de humanas (100\% - 0\%).

Pode-se ressaltar ainda que a autonomia profissional pode estar vinculada ao status profissional e à adoção de postura defensiva frente regalias profissionais, já que os médicos possuem mais autonomia frente a percepção dos profissionais da equipe, assim como possuem um maior status profissional, adotando posturas mais defensivas de acordo com a percepção da equipe.

Os Gráficos 10 e 11 apresentam a percepção dos profissionais da Clínica Pediátrica a respeito da confiança no seu julgamento profissional e da pouca solicitação de conselhos profissionais.

De acordo com os dados, a maioria dos médicos (M), profissionais da área biomédica (B) e dos profissionais da área de humanas $(\mathrm{H})$ confiam no julgamento profissional da equipe, exceto na percepção dos profissionais da área biomédica em relação aos médicos, os quais também julgam que os médicos raramente solicitam seus conselhos profissionais. Tal percepção não está presente na percepção dos médicos (M) e dos profissionais da área de humanas (H), uma vez que os dados evidenciam a existência de trocas profissionais.

Neste caso, evidenciasse que a equipe interdisciplinar vem ganhando cada vez mais espaço no ambiente hospitalar. Como refere Pinho (2006), as instituições estão empenhadas na modificação desse panorama, propiciando aos profissionais uma percepção mais abrangente, dinâmica, complementar e integrada, englobando o modelo biopsicossocial de saúde e, também, a habilidade de estabelecer relações interpessoais de cooperação.

O Gráfico 12 expõe a percepção dos profissionais da Clínica Pediátrica acerca da utilização integral de suas capacidades profissionais.

Os dados do Gráfico 12 demonstram que os médicos e os profissionais da área de humanas percebem que apenas os profissionais da área de humanas da Clínica Pediátrica utilizam integralmente suas capacidades profissionais. Em relação a percepção dos profissionais da área biomédica, a maioria avalia que 
Gráfico 11

Percepção das profissionais da Clínica Pediátrica em relação a pouca solicitação dos conselhos profissionais dentro da equipe

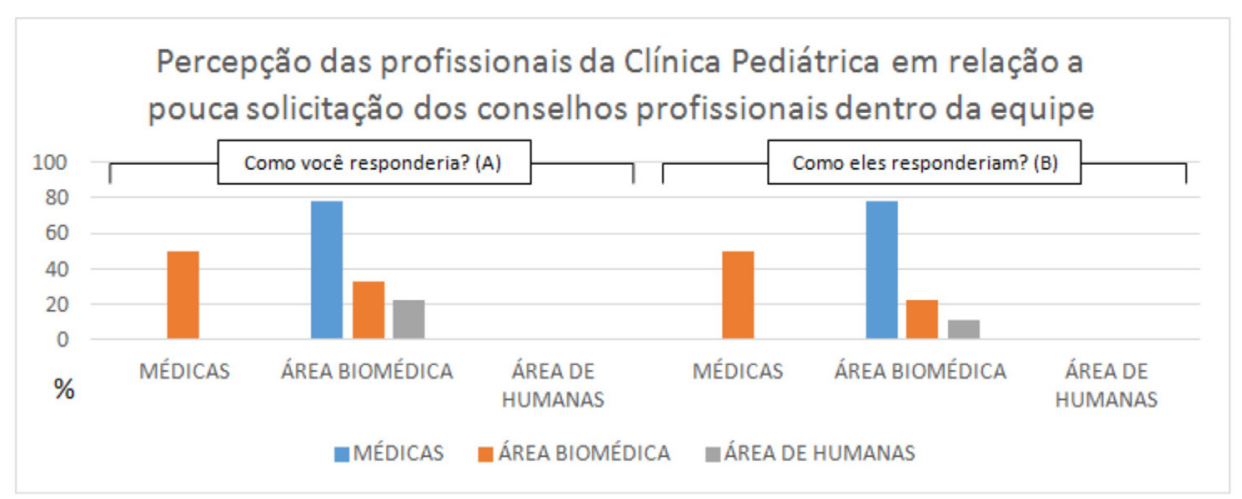

Gráfico 12

Percepção das profissionais da Clínica Pediátrica em relação a utilização integral de suas capacidades profissionais

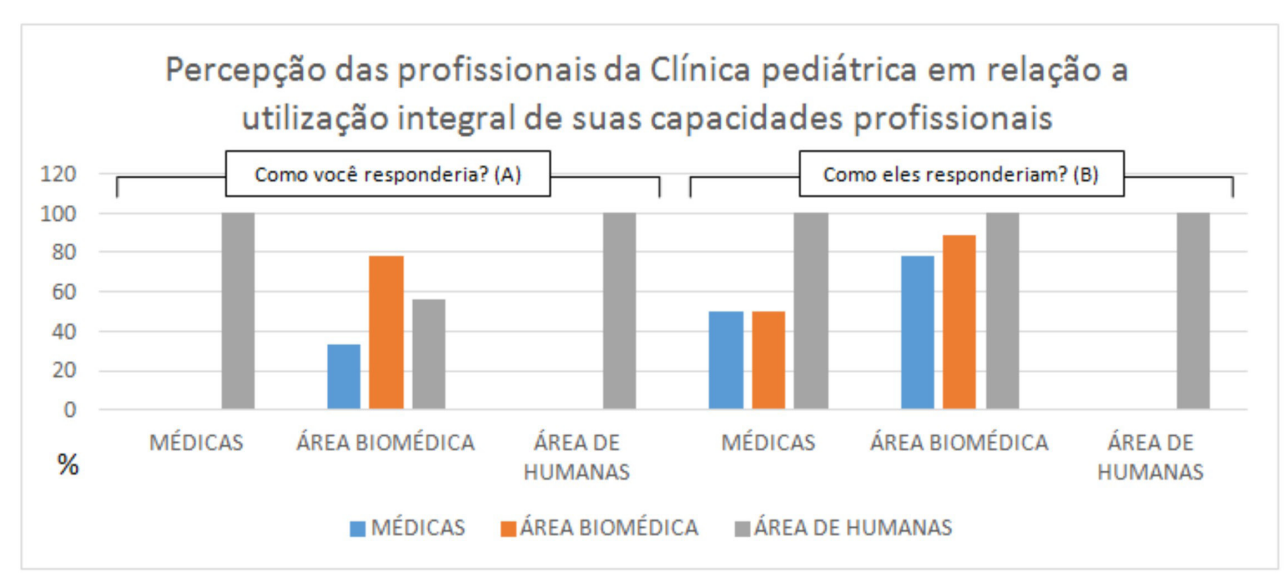

os profissionais da área de biomédicas e de humanas utilizam bem suas capacidades profissionais. Neste caso, pode-se inferir que, principalmente, os médicos precisam fazer melhor uso das suas capacidades profissionais, favorecendo um trabalho em equipe mais abrangente, o que vai ao encontro do que Pinho (2006) ressalta.

Isso pode correr também, como apresenta Santo e Sebastiani (1996), devido a uma formação profissional geradora de sentimentos onipotentes, centralizadores e individualistas, os quais estimulam as barreiras da relação entre os profissionais e, consequentemente, acabam interferindo nas relações da equipe com os pacientes e seus familiares.

Os Gráficos 13,14 e 15 abordam a percepção dos profissionais da Clínica Pediátrica em relação à cooperação entre os profissionais, ao bom treinamento e ao bom relacionamento interprofissional, respectivamente.

Os dados demonstram que a maioria dos profissionais percebem uma boa cooperação profissional em relação aos integrantes da equipe. No entanto, a maioria os profissionais $(56 \%-5)$ da área biomédica avaliam os médicos como pouco cooperadores, havendo ainda uma divergência quando se compara os dois níveis de avaliação A e B $(44 \%-89 \%)$.

Com relação ao bom treinamento (Gráfico 14), os dados apresentam que todos os profissionais médicos e da área de humanas avaliam a equipe como bem treinada, exceto os profissionais médicos em relação aos profissionais biomédicos. A percepção da maioria dos profissionais biomédicos também indica um bom treinamento dentro da equipe de saúde, não havendo divergências significativos entre os dois níveis de avaliação A e B.

Quanto ao bom relacionamento profissional dentro da equipe, o Gráfico 15 aponta que a equipe possui um bom relacionamento interpessoal, salvo na percepção dos profissionais da área biomédica em relação aos médicos, no qual há também uma discrepância quando se compara os dois níveis de avaliação A e B (22\% - 67\%).

Neste sentido, de forma geral e considerando que ainda há dificuldades no trabalho em equipe, a equipe de saúde da Clínica Pediátrica possui cooperação, 
Gráfico 13

Percepção das profissionais da Clínica Pediátrica acerca da cooperação profissional em relação a sua profissão

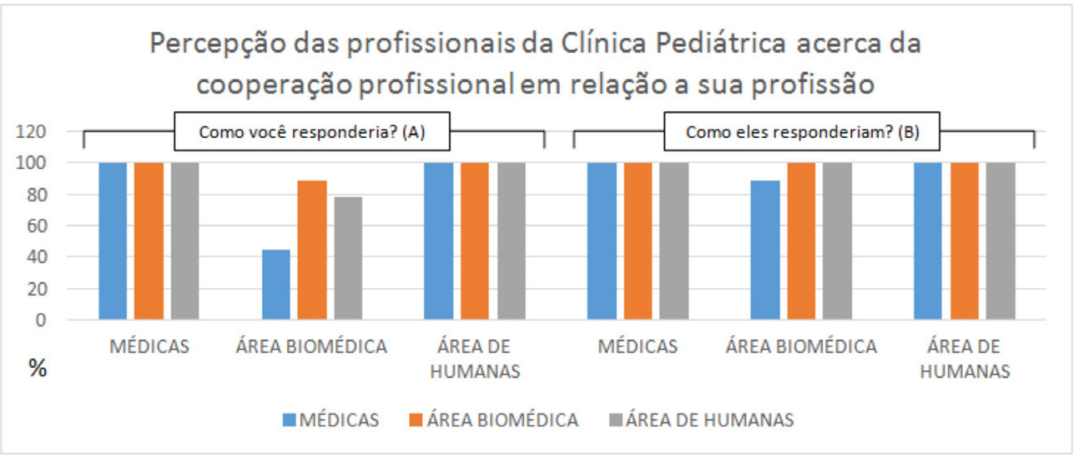

Gráfico 14

Percepção das profissionais da Clínica Pediátrica em relação ao bom treinamento da equipe

Gráfico 15

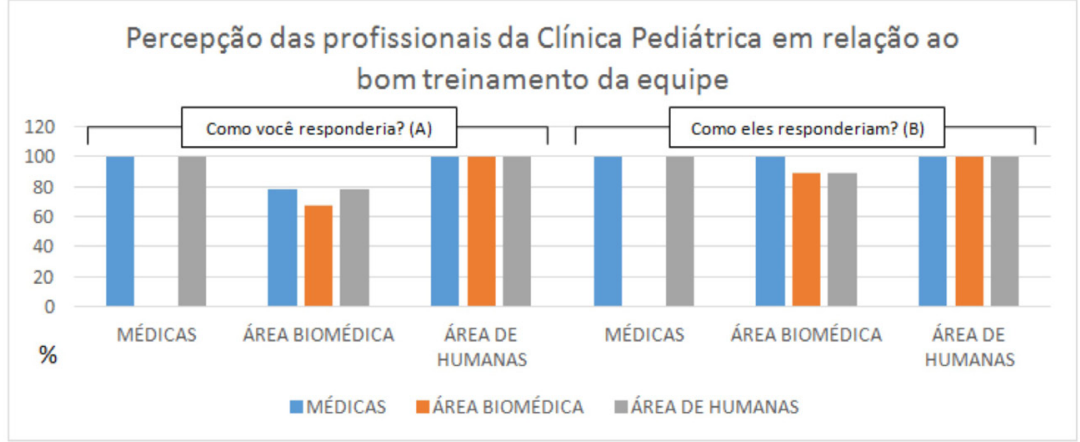

Percepção das profissionais da Clínica Pediátrica acerca do bom relacionamento profissional

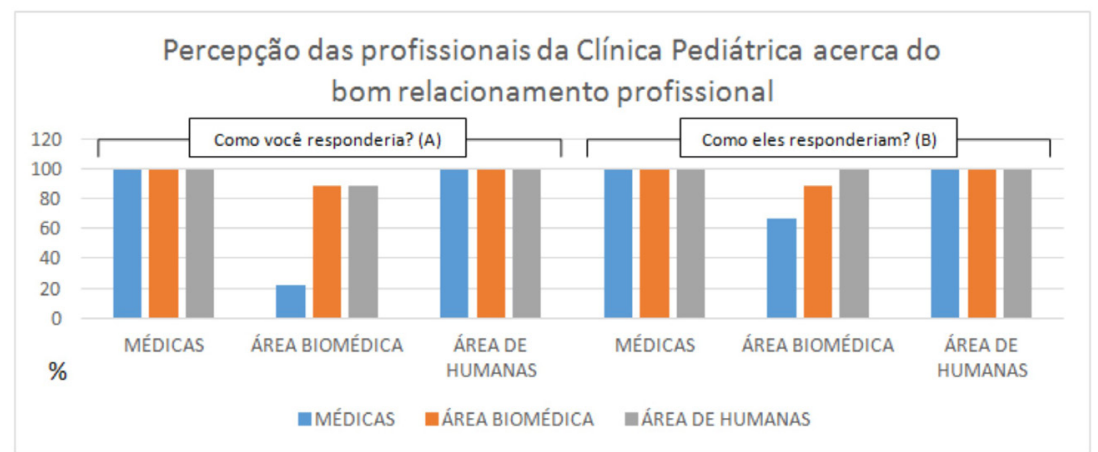

treinamento e relacionamento profissional entre seus membros. Desse modo, Silva, Scapin e Batista (2001) ressaltam que a convivência e a formação interprofissional são capazes de alterar atitudes e percepções negativas entre os membros da equipe de saúde prevenindo falhas na confiança e na comunicação, proporcionando uma melhor integração da equipe.

Partindo do pressuposto que os participantes possuem grau de escolaridade diversificado, há a necessidade de comparação entre as percepções interprofissionais dos profissionais de nível superior e dos profissionais de nível técnico em relação às diversas categorias da clínica pediátrica de um hospital universitário público.

A partir dos dados das percepções interprofissionais de profissionais de nível superior e técnico, pode-se perceber que os profissionais de nível superior apresentaram mais divergências em relação às respostas dos dois níveis de análise do que os profissionais de nível técnico. Este dado pode estar relacionado ao fato dos profissionais de nível técnico atribuírem aos profissionais de nível superior valores associados a domínio de conhecimentos, com funções de caráter mais intelectual, que lhe conferem poder e considerar a si mesmos como executores de tarefas prescritas, de cunho mais manual, como apresentam Souza et al. (2010). 
Tabela 1

Descrição das categorias temáticas de profissionais acerca da caracterização do ambiente profissional e relacional da Clínica Pediátrica de um hospital universitário público

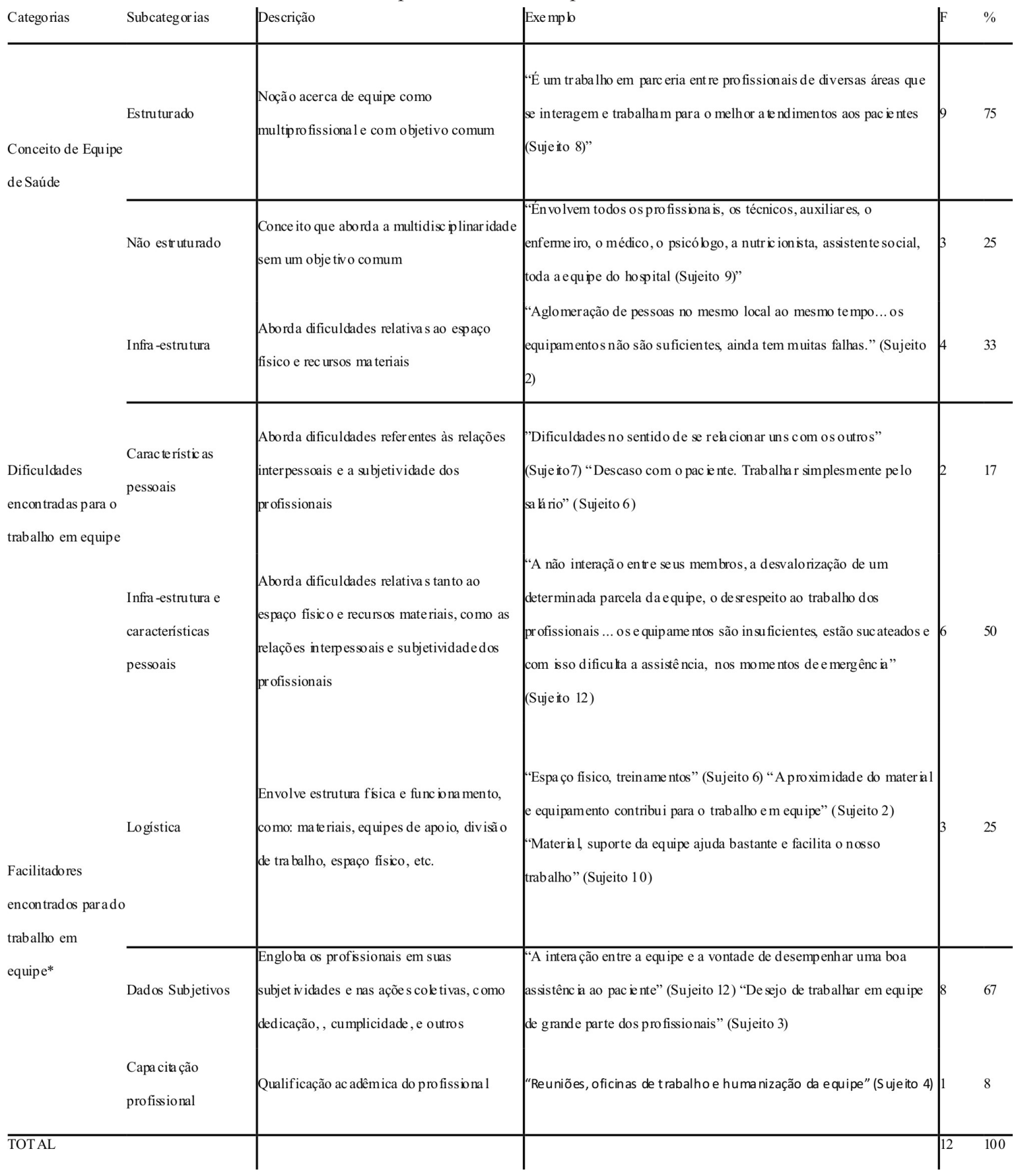

A partir da entrevista realizada com os profissionais da pediatria, os dados coletados deram origem a três categorias de análise referente à equipe de saúde e ao ambiente profissional/relacional da clínica pediátrica de um hospital universitário público, descritas na Tabela 1.

Neste sentido, a primeira categoria de análise na Tabela 1 apresenta que a maioria dos profissionais da equipe de saúde da Clínica Pediátrica de um hospital universitário público, 75\% ( $\mathrm{N}=9)$, apresentou um conceito estruturado e completo de equipe de saúde uma vez que abordaram a multidisciplinaridade visando um fim comum, o bem-estar e a melhoria do atendimento ao paciente. Essa prevalência vai ao encontro do que Abreu et al (2005) apresentam como conceito de equipe, uma vez que os autores a definem como a associação gerencial de habilidade e talentos individuais em uma habilidade coletiva, produzindo atividades e atuações de maneira mais eficiente e efetiva.

No que se refere às dificuldades encontradas para um trabalho em equipe na Clínica Pediátrica de um 
hospital universitário público, os participantes expõem, em sua maioria $(\mathrm{N}=6)$, tanto problemas relacionados ao relacionamento interpessoal como problemas relacionados ao espaço físico e recursos materiais como relevantes. Com isso, pode-se inferir que não é apenas o relacionamento interpessoal que impõe barreiras ao desenvolvimento de um bom trabalho em equipe, já que os recursos materiais e equipamentos, na maioria das vezes sucateados, interferem no bom funcionamento da equipe, imprimindo à mesma um desgaste profissional, aumento do estresse e a necessidade de improvisos frequentes, principalmente em situações de emergência.

Essas barreiras podem estar relacionadas à incipiência da configuração de um trabalho em equipe que, como expõem Queiroz e Araújo (2009), gera muitas limitações associadas ao desconhecimento da atuação e função das demais profissões, supervalorização da própria atuação, formação sem foco no trabalho em equipe, competitividade, falta de confiança e respeitos mútuos, liderança imprópria, excesso de rigidez e déficit na comunicação.

Além disso, corroborando Leite e Vila (2005) expõem que a equipe também vivencia outras situações de muito estresse, como dificuldades frente à morte, à escassez de recursos materiais e humanos, os quais promovem tensões e influenciam negativamente na qualidade do serviço prestado ao usuário.

Em relação à terceira categoria de análise da Tabela 1, a maioria dos profissionais da equipe de saúde da Clínica Pediátrica de um hospital universitário público, 67\% (N=8), alegam que a interação, a proximidade, a humanização, a disposição e o desejo de trabalhar em equipe facilitam e contribuem para o desenvolvimento e qualidade do trabalho prestado pela equipe de saúde da Clínica Pediátrica aos usuários dessa clínica.

Apesar das dificuldades existentes em relação à insuficiência dos recursos materiais e humanos, bem como ao relacionamento interpessoal para o desenvolvimento de um trabalho em equipe na Clínica Pediátrica, esse estudo evidencia que a subjetividade profissional e as ações coletivas facilitam e contribuem para o desenvolvimento e qualidade do trabalho prestado pela equipe de saúde da Clínica Pediátrica aos seus usuários.

Este fator é de extrema importância, como afirmam Santos e Sebastiani (1996), já que as divergências e disputas estimulam os obstáculos na relação equipe-equipe e, consequentemente, acabam influenciando as relações da equipe com os pacientes e seus familiares. Neste sentido, a equipe de saúde da Clínica Pediátrica do hospital universitário público, ao possuir a interação, a proximidade, a humanização, a disposição e o desejo como facilitadores do trabalho em equipe, ressoam, por conseguinte, no apoio à criança e à família, auxiliando no enfrentamento do processo de doença, internação e tratamento.

\section{CONCLUSÃO}

O estudo permitiu a compreensão das percepções interprofissionais dos profissionais da equipe de saúde da pediatria de um hospital universitário público, bem como as barreiras e os facilitadores para o trabalho dessa equipe. Veio demonstrar que apesar da equipe de saúde da Clínica Pediátrica em questão contar com algumas dificuldades relacionadas à falta de recursos materiais e humanos e ao relacionamento interprofissional, bem como constatar algumas divergências nos dois níveis de análise da Escala de Percepção Interprofissional, apresenta uma percepção interprofissional positiva em relação ao funcionamento de sua equipe de saúde.

Apesar dessa percepção positiva, o estudo demonstra a necessidade de estabelecer momentos de reflexão com toda a equipe de saúde da pediatria para a tomada de consciência das dificuldades e facilitadores identificados a respeito da realidade onde a equipe atua, a fim de melhorar a comunicação e o relacionamento entre os profissionais, viabilizando a troca de experiências e conhecimento, o que pode levar a mudanças em direção à integralidade do atendimento e à humanização da assistência prestada aos seus usuários e, consequentemente, melhorar a qualidade do relacionamento interprofissional da equipe de saúde.

\section{REFERÊNCIAS}

Abreu, L. O., Munari, D. B., Queiroz, A. L. B., \& Fernandes, C. N. S. (2005). O trabalho de equipe em enfermagem: revisão sistemática da literatura. Revista Brasileira de Enfermagem, 58 (2), 203-207.

Bardin, L. (2008). Análise de Conteúdo. Lisboa: Edição revista e actualizada. Edições 70.

BRASIL. Ministério da Saúde (1996). Conselho Nacional de Saúde, Comissão Nacional de Ética em Pesquisa. Resolução No 196 , de 10 de outubro de 1996: diretrizes e normas regulamentadoras de pesquisas envolvendo seres humanos. Brasília: Ministério da Saúde do Brasil.

BRASIL. Conselho Nacional de Saúde (1998). Relaciona as categorias profissionais de saúde de nível superior para fins de atuação do Conselho Nacional de Saúde. Resolução N ${ }^{\circ} 287$, de 08 de Outubro de 1998. Brasília: Ministério da Saúde do Brasil.

Castro, E. K. de, \& Piccinini, C. A. (2002). Implicações da doença orgânica crônica na infância para as relações familiares: algumas questões teóricas. Psicologia Reflexão e Crítica, 15 (3), 625-635

Cozby, P. C. (2006). Métodos de pesquisa em ciências do comportamento. São Paulo: Atlas.

Ducanis, A. J.,\& Golin, A. K. (1979). The interdisciplinary health care team. Londom: Aspen System Corporation.

Fossi, L. B., \& Guareschi, N. M. F. (2004). A psicologia hospitalar e as equipes multidisciplinares. Revista SBPH, 7 (1), 29-43.

Galván, G. B. (2007). Equipes de saúde: o desafio da integração disciplinar. Revista SBPH, 10 (2), 53-61.

Leite, M. A., \& Vila, V. S. C. (2005). Dificuldades vivenciadas pela equipe multiprofissional na unidade de terapia intensiva. Revista Latino-Americana de Enfermagem, 13 (2), 145-150.

Mello Filho, J. (1992). Psicossomática hoje. Porto Alegre: 
Artes Médicas

Peduzzi, M. (2001). Equipe multiprofissional de saúde: conceito e tipologia. Revista de Saúde Pública, 35 (1), 103-109.

Pereira, I. M. T. B., \& Harris, W. M. (1976). Estágio integrado na faculdade de saúde pública da universidade de São Paulo: preparo para o trabalho multiprofissional. Revista de Saúde Pública, 10 (3), 257-66.

Pinho, M. C. G. (2006). Trabalho em equipe de saúde: limites e possibilidades de atuação eficaz. Ciência \& Cognição, 08 (1),6887.

Queiroz, E., \& Araújo, T. C. C. F. (2009). Trabalho de equipe em reabilitação: um estudo sobre a percepção individual e grupal dos profissionais de saúde. Paideia, 19 (43), 177-187.

Santos, C. T. dos, \& Sebastiani, R. W. (1996), Acompanhamento psicológico à pessoa portadora de doença crônica. In V. A. Angerami-camon, (Orgs.). E a psicologia entrou no hospital (pp.147-176). São Paulo: Pioneira.

Silva, R. H. A., Scapin, L. T., \& Batista, N. A. (2011), Avaliação da Formação Interprofissional no Ensino Superior em Saúde: aspectos da colaboração e do trabalho em equipe. Revista da Avaliação da Educação Superior, 16 (1), 167-184.

Souza S. S., Costa R., Shiroma L. M. B., Maliska I. C. A., Amadigi F. R., Pires D. E. P. et al. (2010), Reflexões de profissionais de saúde acerca do seu processo de trabalho. Revista Eletrônica de Enfermagem, 12 (3), 449-455.

Vilela, E. M. \& Mendes, I. J. M. (2000), Entre Newton e Einstein: desmedicalizando o conceito de saúde. Ribeirão Preto: Holos.

Wallig, J., \& Filho, E. S. (2007). A psicologia hospitalar segundo médicos e psicólogos: um estudo psicossocial. Cadernos de Psicologia Social do Trabalho, 10 (2), 47-62.

Recebido: 19/03/2013

Última revisão: 12/06/2014

Aceite final: 24/06/2014

Sobre os autores:

Marina Freire Nunes - Graduação em Psicologia pela Universidade Federal de Goiás Pós - Graduação em Psicologia - Residência Multiprofissional em saúde Materno Infantil - Hospital das Clínicas da Universidade Federal de Goiás

Luciana Rodrigues Wovst - Graduação em Psicologia pela Pontifícia Universidade Católica de Goiás - Pós - Graduação em Psicologia - Residência Multiprofissional em saúde Materno Infantil - Hospital das Clínicas da Universidade Federal de Goiás

Sebastião Benício Costa Neto - Hospital das Clínicas da Universidade Federal de Goiás - (Pós) Doutor em Psicologia - UNB 